\title{
Estudo das fontes alimentares de Panstrongylus lutzi (Neiva \& Pinto, 1923) (Hemiptera: Reduviidae: Triatominae) no Estado do Ceará
}

\author{
Feeding sources evaluation of Panstrongylus lutzi (Neiva \& Pinto, 1923) \\ (Hemiptera: Reduviidae: Triatominae) in the State of Ceará
}

\author{
Lindembergh Caranha ${ }^{1}$, Elias Seixas Lorosa ${ }^{2}$, Dayse da Silva Rocha ${ }^{2}$, \\ José Jurberg² e Cleber Galvão ${ }^{2}$
}

\begin{abstract}
RESUMO
Os autores utilizaram a técnica de precipitina para identificar as fontes alimentares de Panstrongylus lutzi (Neiva \& Pinto, 1923) em 20 municípios do Estado do Ceará, Brasil. Os resultados detectaram a presença de sangue de oito diferentes fontes sanguíneas e alimentações mistas, demonstrando que Panstrongylus lutzi é uma espécie eclética. Altas taxas de infecção por tripanosomatídeos foram detectadas.
\end{abstract}

Palavras-chaves: Panstrongylus lutzi. Fontes alimentares. Triatominae. Doença de Chagas.

\begin{abstract}
The authors used precipitin technique to detect the feeding sources of Panstrongylus lutzi (Neiva \& Pinto, 1923) in 20 municipalities of Ceará State, Brazil. The results detected the presence of blood from eight different blood sources and mixed feedings, demonstrating that Panstrongylus lutzi is an eclectic species. High infection rates for Trypanosoma like-cruzi were detected.
\end{abstract}

Key-words: Panstrongylus lutzi. Feeding sources. Triatominae. Chagas' disease.

A doença de Chagas continua sendo um grave problema de saúde pública na América Latina, afetando mais de 12 milhões das pessoas. Os vetores dessa enfermidade são insetos hematófagos da subfamília Triatominae (Hemiptera, Reduviidae) composta atualmente de 136 espécies $^{1011}$. Embora todas as espécies possam ser consideradas como vetores potenciais do Trypanosoma cruzi, apenas algumas delas estão atualmente adaptadas às moradias humanas e, portanto, apresentam importância epidemiológica.

Após o sucesso do controle do Triatoma infestans (Klug, 1834), em países do Cone Sul, e das iniciativas visando controlar populações domésticas de triatomíneos em outros países da América do Sul, o trabalho de vigilância entomológica sobre as espécies com capacidade de invadir e/ou colonizar áreas controladas tornou-se imprescindível. De fato, nos últimos anos, tem ocorrido um incremento do número de relatos de espécies silvestres invadindo as habitações humanas e o peridomicílio no Brasil e países vizinhos 245712131415202224252728 .

Panstrongylus lutzi (Neiva \& Pinto, 1923) é uma das espécies nativas da caatinga, e já foi encontrada em oito estados da região nordeste do Brasil ${ }^{311}$. Pode ser considerada uma das mais importantes dentre aquelas consideradas secundárias na manutenção da doença de Chagas, pois, apresenta altas taxas de infecção natural ${ }^{16}$ e grande capacidade de invasão das residências através do vôo. No Estado do Ceará, sua presença nos domicílios em mais de uma dezena de municípios foi detectada ainda na década de $60^{1}$. No período de 1975 a 1983, foi a oitava espécie mais capturada no Brasil ${ }^{17}$, atingindo à quinta posição em 1999 $9^{26}$; atualmente é encontrada em 137 dos 184 municípios do Estado $^{89}$. Recentemente, sua capacidade de formar pequenas

\footnotetext{
1. Núcleo de Controle de Endemias Transmissíveis por Vetores da Secretaria Estadual de Saúde, Fortaleza, CE. 2. Laboratório Nacional e Internacional de Referência em Taxonomia de Triatomíneos do Instituto Oswaldo Cruz da Fundação Oswaldo Cruz, Rio de Janeiro, RJ.

Trabalho realizado com auxílio do CNPq, Serviço de Vigilância em Saúde - Ministério da Saúde e Chagas Disease Intervention Activities-CDIA Endereço para correspondência: Dr. Cleber Galvão. LNIRTT. Dept ${ }^{\circ}$ de Protozoologia/FIOCRUZ. Av. Brasil 4365, 21040-900 Rio de Janeiro, RJ.

Telefax: $55212560-7317$.

e-mail: galvao@ioc.fiocruz.br

Recebido para publicação em 28/04/2005

Aceito para publicação em 15/5/2006
} 
colônias peridomiciliares no Estado do Ceará foi demonstrada por Sousa \& Galvão $0^{22}$ e Sousa e cols ${ }^{20}$. Apesar de sua crescente importância, pouco se sabe sobre sua biologia e ecologia ${ }^{23}$.

0 estudo dos hábitos alimentares dos triatomíneos através da técnica de precipitina pode contribuir significativamente para ampliar o conhecimento de seus hospedeiros naturais e seu papel na transmissão do T. cruzi, contribuindo para aperfeiçoar o controle e a vigilância dos vetores, uma das metas do programa de controle da doença de Chagas. 0 objetivo do presente trabalho foi identificar as fontes alimentares de espécimes de $P$. lutzi coletados em diversos municípios do Estado do Ceará.

\section{MATERIAL E MÉTODOS}

Os triatomíneos examinados eram adultos e provinham de 20 municípios, onde foram coletados pelos próprios moradores, dentro dos domicílios e encaminhados para os Postos de Identificação de Triatomíneos (PITs) e posteriormente ao laboratório de entomologia médica da $11^{a}$ célula regional da saúde em Sobral. As coletas foram feitas de janeiro a dezembro de 2004. No laboratório, amostras do conteúdo estomacal foram impregnadas em papel de filtro e armazenadas em geladeira até a realização das análises. A identificação das fontes alimentares foi feita utilizando-se a técnica de precipitina ${ }^{19}$. As fontes sangüíneas pesquisadas foram os prováveis hospedeiros que ocorrem na área de estudo. A bateria de anti-soros e os respectivos títulos foram: humano, 1:15.000; ave, 1:14.000; cabra/boi, 1:17.000, cão, 1:15.000; gato, 1:12.000; tatu, 1: 15.000; cavalo,1:15.000; ovelha, 1:13.000; roedor, 1: 15.000, gambá, 1: 10.000, réptil 1: 10.000. Para verificar a infecção por tripanosomatídeos, todos os espécimes foram dissecados com auxílio de pinças entomológicas, e parte do conteúdo estomacal, foi diluído em solução salina. 0 material foi colocado em lâmina para microscopia em camada delgada e examinados em microscópio biológico com aumento de 400X. Posteriormente, todas as lâminas positivas foram fixadas com álcool metílico e coradas pelo método Giemsa-Maygrunwald e examinadas em microscópio biológico com aumentos de 400 e 1000X.

\section{RESULTADOS}

Dos 79 espécimes adultos coletados nos vinte municípios estudados (Figura 1), 63 eram machos e 16 fêmeas (relação 1:4). Não foram encontradas ninfas nos domicílios estudados. Desse total, 23 (29,1\%) estavam positivos para Trypanosoma tipo cruzi. Dentre os municípios estudados, mais de $40 \%$ dos espécimes vieram dos municípios vizinhos, Sobral e Alcântaras (Tabela 1).

Com relação ao estudo das fontes alimentares, das 79 amostras analisadas, 14 (17,7\%) não reagiram, 39 (49,4\%) reagiram para uma única fonte, $23(29,1 \%)$ reagiram para duas fontes enquanto $3(3,8 \%)$ reagiram para três fontes (Tabela 2$)$.
Tabela 1 - Municípios do Estado do Ceará onde foram coletados Panstrongylus lutzi (Neiva \& Pinto, 1923) para o estudo das fontes alimentares.

\begin{tabular}{|c|c|c|}
\hline \multirow[b]{2}{*}{ Município } & \multicolumn{2}{|c|}{ Espécies reagentes } \\
\hline & $\mathrm{n}^{\mathrm{o}}$ & $\%$ \\
\hline Alcântara & 10 & 15,5 \\
\hline Cariré & 8 & 12,4 \\
\hline Frecheirinha & 1 & 1,5 \\
\hline Groairas & 1 & 1,5 \\
\hline Irauçuba & 1 & 1,5 \\
\hline Massapê & 4 & 6,2 \\
\hline Meruoca & 1 & 1,5 \\
\hline Mucambo & 1 & 1,5 \\
\hline Reriutaba & 1 & 1,5 \\
\hline Santana do Acaraú & 9 & 14 \\
\hline Santa Quitéria & 1 & 1,5 \\
\hline Sobral & 14 & 21,6 \\
\hline Cruz & 1 & 1,5 \\
\hline Morrinhos & 6 & 9,3 \\
\hline Carnaubal & 1 & 1,5 \\
\hline Viçosa & 1 & 1,5 \\
\hline Crateús & 1 & 1,5 \\
\hline Ipueiras & 1 & 1,5 \\
\hline Independência & 1 & 1,5 \\
\hline Ipaporanga & 1 & 1,5 \\
\hline Total & 65 & 100,0 \\
\hline
\end{tabular}

Tabela 2 - Fontes alimentares e infecção natural de Panstrongylus lutzi (Neiva \& Pinto, 1923) coletados no Estado do Ceará.

\begin{tabular}{|c|c|c|c|c|}
\hline \multirow[b]{2}{*}{ Fonte alimentar } & \multicolumn{2}{|c|}{ Amostras } & \multicolumn{2}{|c|}{ Positivos para $T$. cruzi } \\
\hline & $\mathrm{n}^{0}$ & $\%$ & $\mathrm{n}^{0}$ & $\%$ \\
\hline$\overline{\text { Ave }}$ & 8 & 10,1 & 0 & 0,0 \\
\hline Roedor & 8 & 10,1 & 2 & 8,6 \\
\hline Gambá & 7 & 8,8 & 6 & 26,0 \\
\hline Boi & 5 & 6,3 & 0 & 0,0 \\
\hline Tatu & 5 & 6,3 & 4 & 17,4 \\
\hline Humano & 3 & 3,8 & 0 & 0,0 \\
\hline Cavalo & 2 & 2,5 & 0 & 0,0 \\
\hline Gato & 1 & 1,3 & 0 & 0,0 \\
\hline Roedor/ave & 6 & 7,6 & 4 & 17,4 \\
\hline Ave/boi & 3 & 3,8 & 0 & 0,0 \\
\hline Boi /roedor & 2 & 2,5 & 1 & 4,4 \\
\hline Tatu/roedor & 2 & 2,5 & 2 & 8,6 \\
\hline Gambá/tatu & 2 & 2,5 & 1 & 4,4 \\
\hline Humano/ave & 1 & 1,3 & 0 & 0,0 \\
\hline Gambá/ave & 1 & 1,3 & 0 & 0,0 \\
\hline Gambá/boi & 1 & 1,3 & 0 & 0,0 \\
\hline Gato/roedor & 1 & 1,3 & 0 & 0,0 \\
\hline Ave/tatu & 1 & 1,3 & 1 & 4,4 \\
\hline Cavalo/ave & 1 & 1,3 & 0 & 0,0 \\
\hline Gato/ave & 1 & 1,3 & 0 & 0,0 \\
\hline Gambá/roedor & 1 & 1,3 & 1 & 4,4 \\
\hline Gambá/ave/boi & 2 & 2,5 & 0 & 0,0 \\
\hline Gambá/ave/roedor & 1 & 1,3 & 1 & 4,4 \\
\hline Não reagentes & 14 & 17,7 & - & - \\
\hline Total & 79 & 100,0 & 23 & 100,0 \\
\hline
\end{tabular}




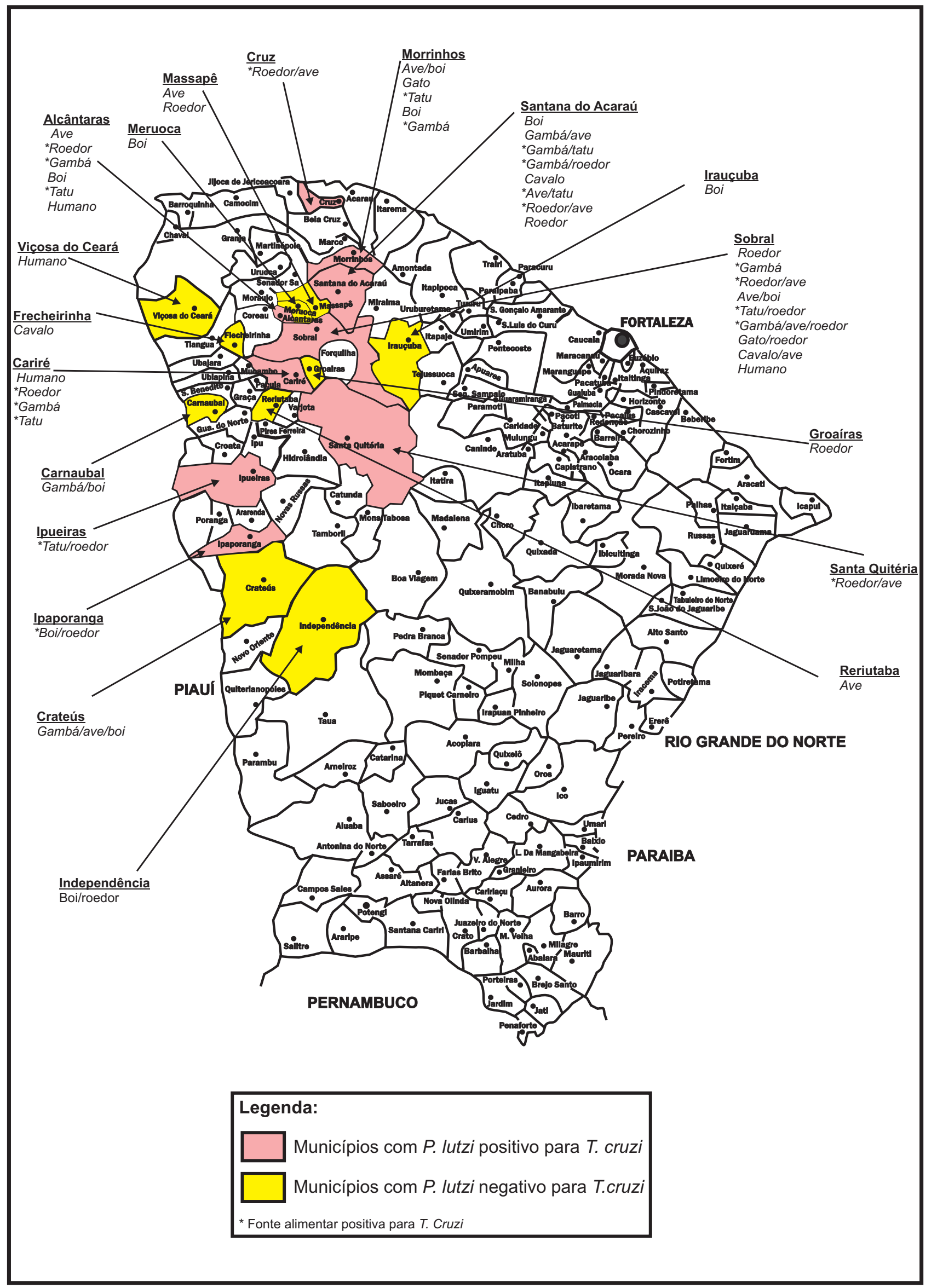

Figura 1- Mapa do Estado do Ceará mostrando a localização dos municípios onde foram coletados espécimes de Panstrongylus lutzi (Neiva \& Pinto, 1923) para estudo de fontes alimentares. 


\section{DISCUSSÃo}

Na região Nordeste do Brasil, a doença de Chagas ocorre de forma endêmica com a presença de espécies nativas responsáveis pela transmissão da doença ao homem. Na atualidade, destacam-se Triatoma brasiliensis Neiva, 1911 e Triatoma pseudomaculata Corrêa \& Espínola, 1964 com ampla distribuição no semi-árido nordestino ${ }^{3}$. Ambas as espécies vem sendo objeto de preocupação e estudos dos órgãos responsáveis pelas ações de controle ${ }^{18}$. Por outro lado, estudos mais aprofundados sobre as espécies emergentes são ainda escassos. No caso de P. lutzi, com exceção dos estudos de Sousa e cols ${ }^{21}$ que encontraram pela primeira vez espécimes sob cascas de Auxemma oncocalyx (Allemao) Taub. (pau branco) e de Dias-Lima e cols ${ }^{6}$ que incriminaram tatus como hospedeiros de P. lutzi, ao encontrarem ninfas dessa espécie em tocas de Dasypodidae, pouco se sabe sobre os habitats e hospedeiros naturais dessa espécie. Os resultados obtidos no presente trabalho mostraram que $P$. lutzi é uma espécie eclética quanto à fonte de alimentação, pois, dentre os espécimes examinados foram observadas alimentações em 8 diferentes fontes. As alimentações mistas sugerem que a espécie circula entre os ambientes silvestre e peridomiciliar, apresentando alta taxa de infecção por Trypanosoma tipo cruzi (29,1\%). A presença desses espécimes no domicílio poderia introduzir cepas silvestres no ambiente domiciliar já que a maioria dos espécimes infectados provavelmente adquiriu a infecção de animais silvestres como, roedores, gambás e tatus. Apesar da presença de cães ser freqüente nos domicílios estudados não foram encontrados espécimes positivos para essa fonte, reforçando a hipótese de que esses insetos se alimentam preferencialmente da fauna silvestre. Por outro lado, é importante ressaltar a participação do homem na cadeia alimentar dessa espécie (4 espécimes), demonstrando ser fundamental a manutenção de um sistema eficiente de vigilância entomológica, aliado à conscientização e educação das populações envolvidas.

\section{REFERÊNCIAS BIBLIOGRÁFICAS}

1. Alencar JE, Sherlock IA. Triatomíneos capturados em domicílios no Estado do Ceará, Brasil. Boletim da Sociedade Cearense de Agronomia 3: 49-54, 1962.

2. Almeida CE, Vinhaes MC, Almeida JR, Silveira AC, Costa J. Monitoring the domiciliary and peridomiciliary invasion process of Triatoma rubrovaria in the state of Rio Grande do Sul, Brazil. Memórias do Instituto Oswaldo Cruz 95: 761-768, 2000.

3. Carcavallo RU, Curto de Casas SI, Sherlock IA, Galíndez-Girón I, Jurberg J, Galvão C, Mena Segura CA. Geographical distribution and altilatitudinal dispersion. In: Carcavallo R, Galíndez-Girón I, Jurberg J, Lent H (orgs) Atlas of Chagas Disease Vectors in the Americas, Vol. III, Editora da Fundação Oswaldo Cruz, Rio de Janeiro, p. 747-792, 1999.

4. Coura JR, Barrett TV, Arboleda MN. Ataque de populações humanas por triatomíneos silvestres no Amazonas: uma nova forma de transmissão da infecção chagásica? Revista da Sociedade Brasileira de Medicina Tropical 27: 251-253, 1994.

5. Coura JR, Junqueira ACV, Bóia MN, Fernandes 0. Chagas disease: from bush to huts and houses. Is it the case of the Brazilian Amazon? Memórias do Instituto Oswaldo Cruz 94: 379-384, 1999.
6. Dias-Lima A, Menezes D, Sherlock I, Noireau F. Wild habitat and related fauna od Panstrongylus lutzi (Reduviidae, Triatominae) Journal Medical Entomology 40: 989-990, 2003.

7. Dias-Lima A, Sherlock I. Sylvatic vectors invading houses and the risk of emergence of cases of Chagas disease in Salvador, state of Bahia, northeast Brazil. Memórias do Instituto Oswaldo Cruz 95: 611-613, 2000.

8. Fundação Nacional de Saúde. Coordenação do Estado do Ceará. Arquivos Estatísticos, Divisão de Epidemiologia, Ministério da Saúde, 1983.

9. Fundação Nacional de Saúde. Coordenação do Estado do Ceará. Arquivos Estatísticos, Divisão de Epidemiologia, Ministério da Saúde, 2001.

10. Forero D, Weirauch C, Baena M. Synonymy of the Reduviidae (Hemiptera: Heteroptera) genus Torrealbaia (Triatominae) with Amphibolus (Harpactorinae), with notes on Amphibolus venator (Klug, 1830). Zootaxa 670:1-12, 2004

11. Galvão C, Carcavallo RU, Rocha DS, Jurberg J. A checklist of the current valid species of the subfamily Triatominae Jeannel, 1919 (Hemiptera, Reduviidae) and their geographical distribution, with nomenclatural and taxonomic notes. Zootaxa 202: 1-36, 2003.

12. Galvão C, Rocha DS, Jurberg J, Carcavallo RU. Ampliação da distribuição geográfica de Triatoma deaneorum Galvão, Souza \& Lima, 1967, nova denominação para Triatoma deanei (Hemiptera, Reduviidae). Revista da Sociedade Brasileira de Medicina Tropical 34: 587-589, 2001.

13. Lorosa ES, Valente MVMP, Cunha V, Lent H, Jurberg J. Foco de doença de Chagas em Arcádia, Estado do Rio de Janeiro. Brasil. Memórias do Instituto Oswaldo Cruz 98: 885-887, 2003.

14. Matias A, De la Riva J, Martinez E, Torrez M, Dujardin JP. Domiciliation process of Rhodnius stali (Hemiptera: Reduviidae) in Alto Beni, La Paz, Bolivia. Tropical Medicine and International Health 8: 264-268, 2003.

15. Sandoval CM, Duarte R, Gutíerrez R, Rocha DS, Ângulo VM, Esteban L, Reyes M, Jurberg J, Galvão C. Feeding sources and natural infection of Belminus herreri (Hemiptera, Reduviidae, Triatominae) from dwellings in Cesar, Colombia. Memórias do Instituto Oswaldo Cruz 99: 137-140, 2004.

16. Silveira AC, Feitosa VR. Altas taxas de infecção natural por Trypanosoma tipo cruzi em Panstrongylus lutzi Neiva \& Pinto, 1923. In: Resumos da XI Reunião Anual de Pesquisa Básica em Doença de Chagas, Caxambu, 1984.

17. Silveira AC, Feitosa VR, Borges R. Distribuição de triatomíneos capturados no ambiente domiciliar, no período de 1975/83, Brasil. Revista Brasileira de Malariologia e Doenças Tropicais 36: 15-312, 1984.

18. Silveira AC, Vinhaes MC, Lira E, Araújo E. O controle de Triatoma brasiliensis e Triatoma pseudomaculata. 1. Estudo do tempo de reposição das condições de transmissão da doença de Chagas por Triatoma brasiliensis e Triatoma pseudomaculata em áreas submetidas a tratamento químico domiciliar, e de variávies ambientais relacionadas. Organização Panamericana da Saúde, Brasília, 2001.

19. Siqueira $\mathrm{AF}$. Estudos sobre a reação da precipitina aplicada à identificação de sangue ingerido por triatomíneos. Revista do Instituto de Medicina Tropical de São Paulo 2: 41-53, 1960.

20. Sousa LC, Bastos, AJ, Sousa AJ, Galvão C. Infestação domiciliar por Panstrongylus lutzi Neiva \& Pinto, 1923 e outras espécies de Triatomíneos (Hemiptera: Reduviidae: Triatominae) na região Noroeste do Ceará. Revista da Sociedade Brasileira de Medicina Tropical 37 (supl 1): 181-182, 2004.

21. Sousa LC, Bezerra FF, Carneiro FFC, Dotaiuti L, Sousa AJ. Descrição de um ecótopo natural do Panstrongylus lutzi Neiva \& Pinto, 1923 (Hemiptera: Reduviidae: Triatominae) em Sobral, Norte do Ceará. Revista da Sociedade Brasileira de Medicina Tropical 36 (supl 1): 26, 2003.

22. Sousa LC, Galvão C. Colonização do Panstrongylus lutzi Neiva \& Pinto, 1923 (Hemiptera: Reduviidae: Triatominae) em ecótopos artificiais no Ceará. Revista da Sociedade Brasileira de Medicina Tropical 37 (supl 1): 35, 2004.

23. Sousa LC, Lorosa ES, Rocha DS, Jurberg J, Galvão C. Estudo das fontes alimentares de Panstrongylus lutzi (Neiva \& Pinto, 1923) (Hemiptera: Reduviidae: Triatominae) no Estado do Ceará. Revista da Sociedade Brasileira de Medicina Tropical 38 (supl 1): 56, 2005.

24. Valente VC. Potential for domestication of Panstrongylus geniculatus (Latreille, 1811) (Liemiptera, Reduviidae, Triatominae) in the municipality 
of Muaná, Marajó Island, State of Pará, Brazil. Memórias do Instituto Oswaldo Cruz 94: 399-400, 1999.

25. Valente VC, Valente SAS, Noireau F, Carrasco HJ, Miles M. Chagas disease in the Amazon Basin: association of Panstrongylus geniculatus (Hemiptera: Reduviidae) with domestic pigs. Journal Medical Entomology 35: 99-103, 1998.

26. Vinhaes MC, Dias JCP. Doença de Chagas no Brasil. Cadernos de Saúde Pública 16 (supl 2): 7-12, 2000.
27. Vivas AS, Barazarte H, Molina de Fernandéz D. Primer registro de Eratyrus mucronatus Stål, 1959 (Hemiptera: Reduviidae) en el ambiente domiciliário em Venezuela. Entomotropica 16: 215-217, 2001.

28. Wolff M, Castillo D. Domiciliation trend of Panstrongylus rufotuberculatus in Colombia. Memórias do Instituto Oswaldo Cruz 97: 297-300, 2002. 